\title{
Minimizing Harmonic Distortion Impact at Distribution System with Considering Large-Scale EV Load Behaviour Using Modified Lightning Search Algorithm and Pareto-Fuzzy Approach
}

\author{
S. N. Syed Nasir $\mathbb{D}$, J. J. Jamian $(\mathbb{D}$, and M. W. Mustafa $\mathbb{E}$ \\ Faculty of Electrical Engineering, Universiti Teknologi Malaysia (UTM), 81310 Johor Bahru, Johor, Malaysia \\ Correspondence should be addressed to J. J. Jamian; jasrul@fke.utm.my
}

Received 22 October 2017; Accepted 4 January 2018; Published 15 February 2018

Academic Editor: Fernando Lezama

Copyright (C) 2018 S. N. Syed Nasir et al. This is an open access article distributed under the Creative Commons Attribution License, which permits unrestricted use, distribution, and reproduction in any medium, provided the original work is properly cited.

\begin{abstract}
This research is focusing on optimal placement and sizing of multiple variable passive filter (VPF) to mitigate harmonic distortion due to charging station (CS) at 449 bus distribution network. There are 132 units of CS which are scheduled based on user behaviour within 24 hours, with the interval of 15 minutes. By considering the varying of CS patterns and harmonic impact, Modified Lightning Search Algorithm (MLSA) is used to find 22 units of VPF coordination, so that less harmonics will be injected from $415 \mathrm{~V}$ bus to the medium voltage network and power loss is also reduced. Power system harmonic flow, VPF, CS, battery, and the analysis will be modelled in MATLAB/m-file platform. High Performance Computing (HPC) is used to make simulation faster. Pareto-Fuzzy technique is used to obtain sizing of VPF from all nondominated solutions. From the result, the optimal placements and sizes of VPF are able to reduce the maximum THD for voltage and current and also the total apparent losses up to $39.14 \%, 52.5 \%$, and $2.96 \%$, respectively. Therefore, it can be concluded that the MLSA is suitable method to mitigate harmonic and it is beneficial in minimizing the impact of aggressive CS installation at distribution network.
\end{abstract}

\section{Introduction}

The vision to have less carbon dioxide (CO2) emissions and less dependency on natural resources has encouraged Electric Vehicle (EV) to become an important option compared to conventional vehicle. Based on report in 2015, around $56.4 \%$ global crude oil is used for transportation sector [1]. Furthermore, the unstable price for crude oil has also influenced EV to become suitable alternative. The demand on EV, indirectly, has increased the number of CS installation in the distribution system [2]. However, power losses will increase when a large number of unplanned CS are installed. Furthermore, it will also introduce harmonic distortion due to the power electronic devices that convert alternating current (AC) to direct current (DC) at CS [3, 4]. This harmonic distortion will cause negative impact such as increment heating losses, shorter insulation lifespan, increased temperature and insulation stress, decreased power factor, and lower efficiency $[5,6]$.
Currently, most of the researchers focus on strategies to reduce loss by coordinating the charging of EVs. Alonso et al. [7] have developed an optimization algorithm to coordinate the charging of EVs using Genetic Algorithm (GA). The charging schedule is based on optimal load pattern for EV charging-based reliability which takes into consideration the thermal line limits, the load on transformers, voltage limits, and parking availability patterns. The proposed method is able to reduce the cost for power system new investment. Furthermore, Bharati and Paudyal [8] have proposed framework to coordinate EVs in the distribution system using bilevel hierarchical vehicle-grid (VG) optimization. This framework requires information exchange among $\mathrm{EV}$ aggregators and grid controller and then the EV charging can be scheduled based on the minimum losses to the distribution network. Next, Masoum and Nabavi [9] have also proposed onlineovernight PEV coordination using metaheuristic technique which is particle swarm optimization. This method introduces high priority and low priority customer that indicate 


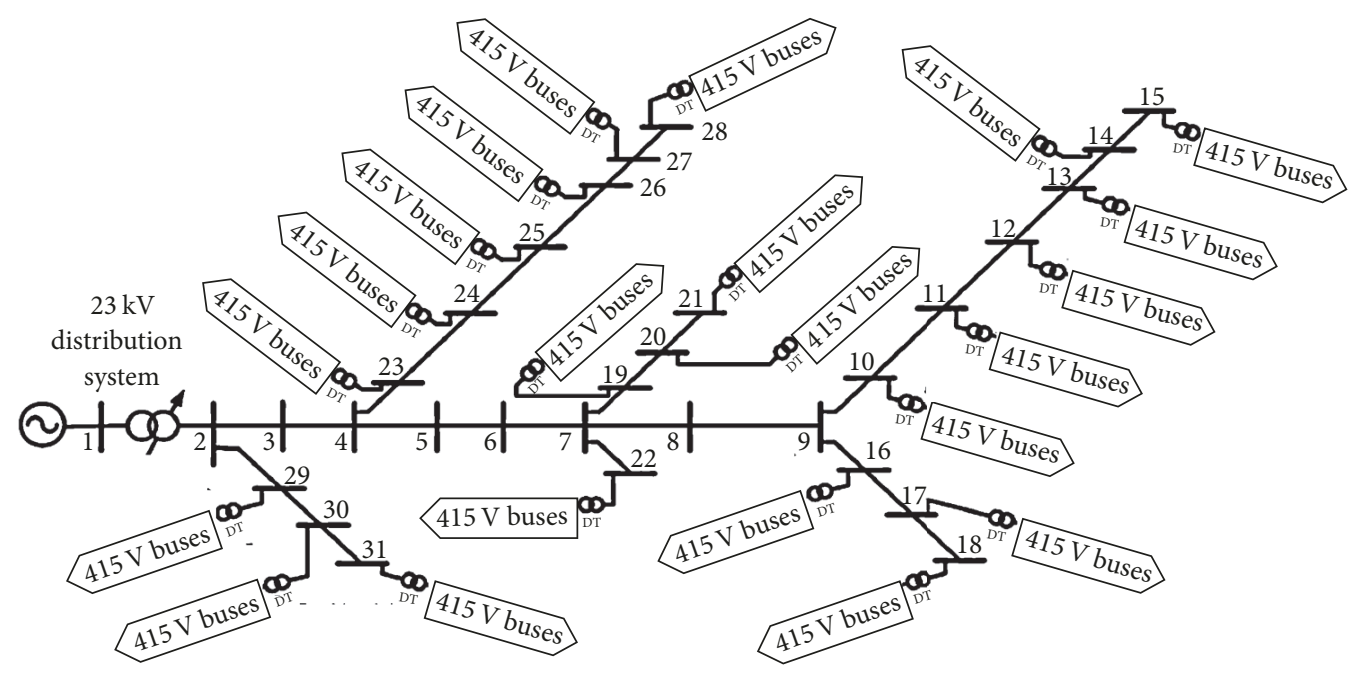

FIGURE 1: Single-line diagram for 449 bus radial distribution system.

the willingness of customer to be charged. High priority customer will get faster charging services with high tariff and the low priority customer will only charge their vehicle after power loss control in the distribution system is done. Even though many approaches have been introduced to minimize the EV impact, the focus is more towards minimizing the power loss. However, beside power loss, the harmonic injection to the distribution system when many CS are connected must also be taken into consideration in ensuring that the network can operate optimally.

In general, there will be two types of harmonic that will be mitigated in distribution network, which are voltage and current harmonic. Voltage harmonic is measured at the bus, while current harmonic is measured at the lines and cables. Passive filter is the common component used to eliminate these harmonics. Passive filters can provide a low-impedance path to harmonic currents and hence prevent it from flowing into the systems. There are many papers that utilized passive filter to mitigate harmonics in power system using filter devices [10-14] and the most common ones are single tuned filters. Single tuned filters are designed to eliminate or reduce single frequency from the system depending on the design resistance, capacitance, and inductance value [15]. Sakar et al. [16] have suggested a hosting capacity determination for a distorted distribution system due to Photovoltaic connection. In their research, passive filter is used to increase the harmonic-constrained hosting capacity which then improves the voltage, power factor, and filtering the harmonics. Author in [17] proposed an Asymmetric Synchronous Reference Frame Control scheme and harmonic voltage compensator with a Static Var Compensator connected to the grid to mitigate the harmonic. The author used harmonic voltage compensator to reduce voltage THD. Even though many researchers have used passive filter to mitigate the harmonic, very few researchers considered both voltage and current harmonics in their analysis especially for CS.

Therefore, this research proposes a coordination of 22 VPF when 132 unit of CS is adopted in a 449 bus radial

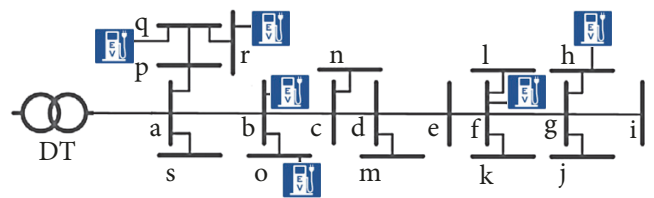

FIGURE 2: Single-line diagram for $415 \mathrm{~V}$ buses.

distribution system. Section 2 presents the modelling of load profile, CS, battery, passive filter, harmonic, CS scheduling, and radial load flow used in this study. The introduction of MLSA technique will also be covered in this section. The methodology of the proposed coordination will be discussed clearly in Section 3. Result and discussion for 22 units of filter coordination in 449 bus are presented in Section 4. Lastly, conclusion of this research is in Section 5.

\section{Problem Modelling and Formulation}

A typical 449 bus radial distribution system is used in this research to determine the optimal placement and sizing of VPF. Furthermore, forward/backward sweep method is chosen due to the accuracy of this load flow analysis in solving distribution load flow [18-21]. The harmonic pattern for individual EV charger is modelled based on actual single phase charger impact as measured in [22].

2.1. IEEE 31 Bus with 22 Low Voltage $415 \mathrm{~V}$ Bus System. Figures 1 and 2 show a single-line diagram for $23 \mathrm{kV}$ IEEE 31 bus radial system with low voltage $415 \mathrm{~V}$ buses residential feeder that consist of several CS, respectively. The total number of buses in the network (medium and low voltages) is 449 buses. There are 6 units of CS installed at one residential area which add up to 132 units for overall CS in this distribution system. Line data for low voltage $415 \mathrm{~V}$ buses is shown in Table 1.

2.2. Harmonic Load Flow Analysis. Many techniques can be used to perform harmonic analysis such as in [23-25] and the 
TABLE 1: Line data for $415 \mathrm{~V}$ buses.

\begin{tabular}{lccc}
\hline \multicolumn{2}{c}{ Line } & Line resistance $R(\Omega)$ & Line reactance $X(\Omega)$ \\
Bus A & Bus B & & \\
\hline a & $\mathrm{b}$ & 0.0415 & 0.0145 \\
$\mathrm{~b}$ & $\mathrm{c}$ & 0.0424 & 0.0189 \\
$\mathrm{c}$ & $\mathrm{d}$ & 0.0444 & 0.0198 \\
$\mathrm{~d}$ & $\mathrm{e}$ & 0.0369 & 0.0165 \\
$\mathrm{e}$ & $\mathrm{f}$ & 0.0520 & 0.0232 \\
$\mathrm{f}$ & $\mathrm{g}$ & 0.0524 & 0.0234 \\
$\mathrm{~g}$ & $\mathrm{~h}$ & 0.0005 & 0.0002 \\
$\mathrm{~g}$ & $\mathrm{i}$ & 0.2002 & 0.0199 \\
$\mathrm{~g}$ & $\mathrm{j}$ & 1.7340 & 0.1729 \\
$\mathrm{f}$ & $\mathrm{k}$ & 0.2607 & 0.0260 \\
$\mathrm{f}$ & $\mathrm{l}$ & 1.3605 & 0.1357 \\
$\mathrm{~d}$ & $\mathrm{~m}$ & 0.1400 & 0.0140 \\
$\mathrm{c}$ & $\mathrm{n}$ & 0.7763 & 0.0774 \\
$\mathrm{~b}$ & $\mathrm{o}$ & 0.5977 & 0.0596 \\
$\mathrm{a}$ & $\mathrm{p}$ & 0.1423 & 0.0496 \\
$\mathrm{p}$ & $\mathrm{q}$ & 0.0837 & 0.0292 \\
$\mathrm{q}$ & $\mathrm{r}$ & 0.3123 & 0.0311 \\
$\mathrm{a}$ & $\mathrm{s}$ & 0.0163 & 0.0062 \\
DT & & 0.0000 & 0.0654 \\
\hline
\end{tabular}

most typical and simplest one is by using current injection analysis method [26]. The electrical parameters that affected the harmonic existence are the line impedances, load variation impedances, and filter impedances values. Equations (1) and (2) represent the line impedance formulation and the impedance for single-tuned filter at harmonic $h$, respectively, which indirectly caters for resonance impact. Equations (3), (4), and (5) show the impedance formulation for the load used in setting up the harmonic admittance matrix. The harmonic flow is calculated using (6), which consists of harmonic admittance matrix and harmonic current injection as per (7) and (8).

$$
\begin{aligned}
Z_{l, h} & =R_{l}+j h \omega L_{l}, \\
Z_{\text {Filter }, h} & =R_{\text {Filter }}+j h \omega L_{\text {Filter }}-j \frac{1}{h \omega C_{\text {Filter }}}, \\
Z_{L, h} & =R_{L}+j X_{L, h}, \\
R_{L} & =\frac{V_{L}^{2}}{P_{L}} \\
X_{L, h} & =\frac{V_{L}^{2}}{h Q_{L}}, \\
\bar{I}_{h} & =\bar{Y}_{h} \cdot \bar{V}_{h}, \\
\bar{Y}_{h} & =\left[\begin{array}{cccc}
\bar{y}_{11, h} & \bar{y}_{12, h} & \cdots & \bar{y}_{1 N, h} \\
\bar{y}_{21, h} & \bar{y}_{22, h} & \cdots & \vdots \\
\vdots & \vdots & \cdots & \vdots \\
\bar{y}_{N 1, h} & \cdots & \cdots & \bar{y}_{N N, h}
\end{array}\right],
\end{aligned}
$$

$$
\bar{I}_{h}=\left[\begin{array}{c}
\bar{I}_{1, h} \\
\bar{I}_{2, h} \\
\vdots \\
\bar{I}_{N, h}
\end{array}\right] .
$$

2.3. Modelling of CS and Battery. CS is typically divided into three categories which are level 1, level 2, and level 3. The level of the CS is based on the power consumed by the charging station. Level 1 takes 6-8 hours, while level 3 requires 30 minutes for fully charging the EV's battery. In this research, level 1 CS is selected since this research focuses on residential area which normally involves single-phase supply. The main different in this work is on CS modelling and its behaviour. The CS modelling in this research has considered the worst harmonic value achievable by individual CS based on the battery characteristic and State of Charge (SOC) state. The initial and final (plug-in and plug-out) battery SOC value depended on customer request, while the current produced by CS to the EV battery is calculated based on that SOC state. SOC state will be updated in every 15 minutes $(\Delta t)$ and can be calculated using (9), while the current produced by CS can be calculated using (10).

$$
\begin{aligned}
& \operatorname{SOC}\left(\Delta t_{k+1}, i\right)=\operatorname{SOC}\left(\Delta t_{k}, i\right)+\left(\frac{\Delta t}{Q_{i}} I\left(\Delta t_{k}, i\right)\right) \times 100, \\
& I\left(\Delta t_{k}, i\right) \\
& =\frac{\sqrt{\left(4 \cdot \Delta t \cdot R_{i} \cdot P_{\mathrm{CS}}\left(\Delta t_{k}, i\right) \cdot \eta \cdot \mathrm{CR}_{i\left(\Delta t_{k}, i\right)}\right)+V_{\mathrm{oc}, i}^{2}}-V_{\mathrm{oc}, i}}{2 R_{i} \cdot \mathrm{CR}_{i\left(\Delta t_{k}, i\right)}},
\end{aligned}
$$

where $V_{\mathrm{oc}, i}$ is open circuit voltage for th node $(\mathrm{V}), Q_{i}$ is rated battery ampere hour for the ith PEV (Ah), $R_{i}$ is battery equivalent internal resistance for the $i$ th node (ohm), $\mathrm{CR}_{i\left(\Delta t_{k}, i\right)}$ is maximum charging rate for the $i$ th $\operatorname{PEV~(A),~}$ $\operatorname{SOC}\left(\Delta t_{k}, i\right)$ is state of charge of the $i$ th PEV at $k$ th time slot (\%), $\operatorname{SOC}\left(\Delta t_{k+1}, i\right)$ is state of charge of the $i$ th PEV at next $k$ th time slot $(\%), I\left(\Delta t_{k}, i\right)$ is charging current for the $i$ th PEV at current time slot $(\mathrm{A})$, and $P_{\mathrm{CS}}\left(\Delta t_{k}, i\right)$ is consumed power for the $i$ th PEV $(\mathrm{kW})$.

Figure 3 shows the open circuit voltage (OCV) for lithium iron phosphate (LFP) batteries based on the SOC of battery [27] that can also be presented using (11). Thus, the relation between SOC and $V_{\mathrm{OCV}}$ will influence the total current injected during charging process.

$$
\begin{aligned}
V_{\mathrm{OCV}}= & 3.135-0.685(-\ln (\mathrm{SOC}))^{0.478} \\
& -(1.342 \cdot \mathrm{SOC})+\left(1.734 \cdot e^{0.4(\mathrm{SOC}-1)}\right) .
\end{aligned}
$$

Since there is conversion from AC to DC during charging process, the harmonic impact to the network is considered in this study. The harmonic injection is basically based on SOC state, which depended on battery SOC status and is being modelled based on practical data from [18]. Since there are 132 CS units installed within the 22 low voltage residential areas (6 units for every residential area) it might have significant 
TABLE 2: CS and battery data.

\begin{tabular}{lcccc}
\hline Type & Battery capacity $(\mathrm{kW})$ & Charging rate $(\mathrm{A})$ & Power usage $(\mathrm{kW})$ & CS efficiency \\
\hline 1 & 10 & 0.052 & 0.52 & 0.93 \\
2 & 15 & 0.125 & 2.875 & 0.93 \\
3 & 10 & 0.235 & 2.35 & 0.93 \\
4 & 15 & 0.15 & 25 & 0.93 \\
5 & 10 & 0.215 & 2.15 & 0.93 \\
6 & 20 & 0.102 & 2.04 & 0.93 \\
\hline
\end{tabular}

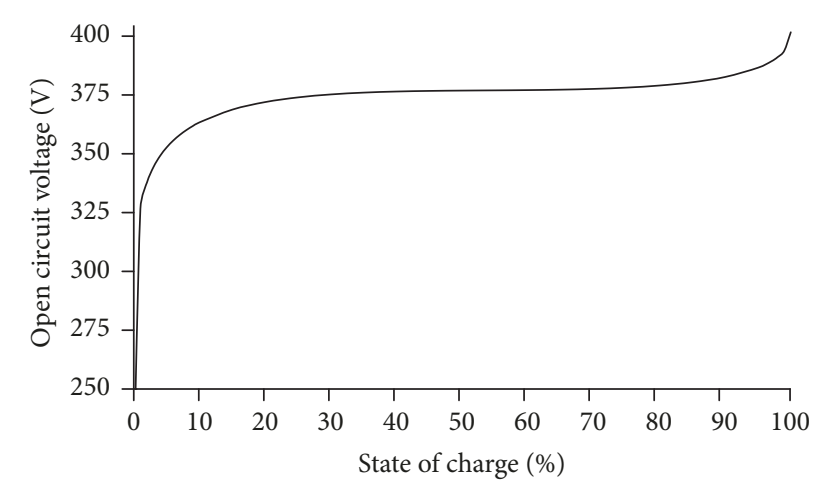

FIGURE 3: Relationship between open circuit voltage and SOC for LFP battery.

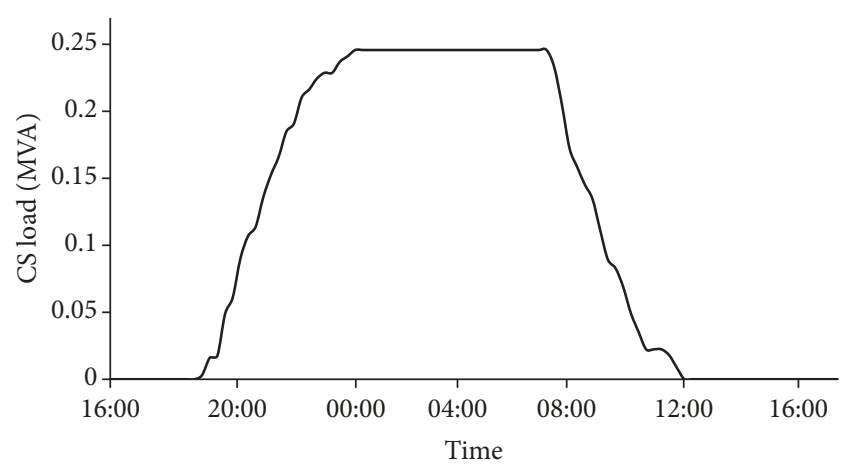

FIGURE 4: Load produced by CS over time.

impact on $\mathrm{THD}_{V}$ and $\mathrm{THD}_{i}$ value when all CS operated simultaneously. All CS and battery data in the residential area are presented as per Table 2 .

\subsection{Modelling of Load Profile and Charging Station Operation.} The load profile for 449 bus system is design based on typical load consumption in residential area during normal day. The peak demand in the system is occurring at the afternoon due to the several usages of electrical appliances, while the lowest demand is at the night when majority of people are asleep. However, the new electrical load CS is normally being charged at the night time. In this study, it is assumed that harmonic produced in the distribution system is coming from CS. Figure 4 shows the loading produced by all CS in the distribution system based on design of CS operation as in Table 3. CS operation is designed based

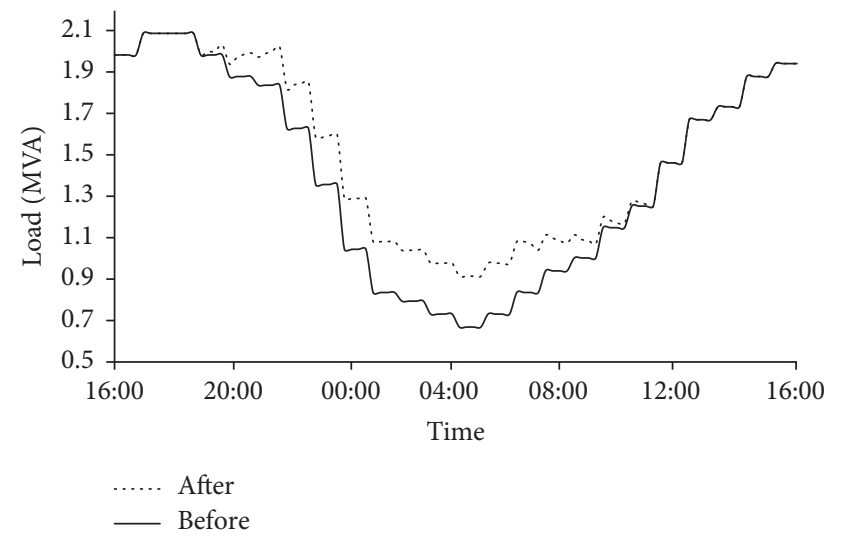

FIGURE 5: Load profile before and after CS operation.

on random behaviour and will penetrate at the night hour. Figure 5 shows the summation of original load profile with CS load. Even though most of the CS is operating during low loaded condition at distribution system, it will cause higher impact on the total harmonic distortion as well as power losses.

2.5. Assumptions and Constraints. There are few assumptions and constraints are considered in this research as shown below:

(i) CS can be plugged in/plugged out at any time according to the customer's request. Customers will input their requested plug-out time and requested final SOCs at the time of plug-in. Once SOC reaches requested SOC, CS will be switched to a standby mode.

(ii) The time slot is $15 \mathrm{~min}(\Delta t)$ or equal to 96 slots for one day.

(iii) The aggregator has access to CS information including their bus locations, harmonic distortion information, charger types, battery sizes, plug-in time, and plug-out time.

(iv) CS are controllable and have variable charging functions. During the charging process, each CS is assumed as a variable active load. The power that is used to charge the battery is based on calculation that is done by the aggregator. 
TABle 3

\begin{tabular}{|c|c|c|c|c|c|}
\hline Bus & Plug-in & $\begin{array}{c}\text { Plug- } \\
\text { out }\end{array}$ & $\begin{array}{c}\text { SOC } \\
\text { initial }\end{array}$ & $\begin{array}{c}\text { SOC } \\
\text { Req }\end{array}$ & Type \\
\hline 33 & 18 & 64 & 28 & 58 & 1 \\
\hline 37 & 21 & 64 & 11 & 80 & 2 \\
\hline 39 & 23 & 65 & 10 & 82 & 3 \\
\hline 46 & 21 & 64 & 1 & 78 & 4 \\
\hline 48 & 19 & 63 & 1 & 69 & 5 \\
\hline 49 & 22 & 65 & 4 & 85 & 6 \\
\hline 52 & 15 & 61 & 28 & 58 & 1 \\
\hline 56 & 18 & 61 & 11 & 80 & 2 \\
\hline 58 & 20 & 62 & 10 & 82 & 3 \\
\hline 65 & 18 & 61 & 1 & 78 & 4 \\
\hline 67 & 16 & 60 & 1 & 69 & 5 \\
\hline 68 & 19 & 62 & 4 & 85 & 6 \\
\hline 71 & 23 & 69 & 28 & 58 & 1 \\
\hline 75 & 26 & 69 & 11 & 80 & 2 \\
\hline 77 & 28 & 70 & 10 & 82 & 3 \\
\hline 84 & 26 & 69 & 1 & 78 & 4 \\
\hline 86 & 24 & 68 & 1 & 69 & 5 \\
\hline 87 & 27 & 70 & 4 & 85 & 6 \\
\hline 90 & 13 & 59 & 28 & 58 & 1 \\
\hline 94 & 16 & 59 & 11 & 80 & 2 \\
\hline 96 & 18 & 60 & 10 & 82 & 3 \\
\hline 103 & 16 & 59 & 1 & 78 & 4 \\
\hline 105 & 14 & 58 & 1 & 69 & 5 \\
\hline 106 & 17 & 60 & 4 & 85 & 6 \\
\hline 109 & 17 & 60 & 4 & 85 & 6 \\
\hline 113 & 14 & 58 & 1 & 69 & 5 \\
\hline 115 & 16 & 59 & 1 & 78 & 4 \\
\hline 122 & 18 & 60 & 10 & 82 & 3 \\
\hline 124 & 16 & 59 & 11 & 80 & 2 \\
\hline 125 & 13 & 59 & 28 & 58 & 1 \\
\hline 128 & 26 & 69 & 4 & 85 & 6 \\
\hline 132 & 23 & 67 & 1 & 69 & 5 \\
\hline 134 & 25 & 78 & 1 & 78 & 4 \\
\hline 141 & 27 & 82 & 10 & 82 & 3 \\
\hline 143 & 25 & 80 & 11 & 80 & 2 \\
\hline 144 & 22 & 58 & 28 & 58 & 1 \\
\hline 147 & 33 & 76 & 4 & 85 & 6 \\
\hline 151 & 30 & 74 & 1 & 69 & 5 \\
\hline 153 & 32 & 75 & 1 & 78 & 4 \\
\hline 160 & 34 & 76 & 10 & 82 & 3 \\
\hline 162 & 32 & 75 & 11 & 80 & 2 \\
\hline 163 & 29 & 75 & 28 & 58 & 1 \\
\hline 166 & 18 & 64 & 28 & 58 & 1 \\
\hline 170 & 21 & 64 & 11 & 80 & 2 \\
\hline 172 & 23 & 65 & 10 & 82 & 3 \\
\hline 179 & 21 & 64 & 1 & 78 & 4 \\
\hline 181 & 19 & 63 & 1 & 69 & 5 \\
\hline 182 & 22 & 65 & 4 & 85 & 6 \\
\hline
\end{tabular}

TABle 3: Continued.

\begin{tabular}{|c|c|c|c|c|c|}
\hline Bus & Plug-in & $\begin{array}{c}\text { Plug- } \\
\text { out }\end{array}$ & $\begin{array}{c}\text { SOC } \\
\text { initial }\end{array}$ & $\begin{array}{c}\text { SOC } \\
\text { Req }\end{array}$ & Type \\
\hline 185 & 15 & 61 & 28 & 58 & 1 \\
\hline 189 & 18 & 61 & 11 & 80 & 2 \\
\hline 191 & 20 & 62 & 10 & 82 & 3 \\
\hline 198 & 18 & 61 & 1 & 78 & 4 \\
\hline 200 & 16 & 60 & 1 & 69 & 5 \\
\hline 201 & 19 & 62 & 4 & 85 & 6 \\
\hline 204 & 23 & 69 & 28 & 58 & 1 \\
\hline 208 & 26 & 69 & 11 & 80 & 2 \\
\hline 210 & 28 & 70 & 10 & 82 & 3 \\
\hline 217 & 26 & 69 & 1 & 78 & 4 \\
\hline 219 & 24 & 68 & 1 & 69 & 5 \\
\hline 220 & 27 & 70 & 4 & 85 & 6 \\
\hline 223 & 13 & 59 & 28 & 58 & 1 \\
\hline 227 & 16 & 59 & 11 & 80 & 2 \\
\hline 229 & 18 & 60 & 10 & 82 & 3 \\
\hline 236 & 16 & 59 & 1 & 78 & 4 \\
\hline 238 & 14 & 58 & 1 & 69 & 5 \\
\hline 239 & 17 & 60 & 4 & 85 & 6 \\
\hline 242 & 17 & 60 & 4 & 85 & 6 \\
\hline 246 & 14 & 58 & 1 & 69 & 5 \\
\hline 248 & 16 & 59 & 1 & 78 & 4 \\
\hline 255 & 18 & 60 & 10 & 82 & 3 \\
\hline 257 & 16 & 59 & 11 & 80 & 2 \\
\hline 258 & 13 & 59 & 28 & 58 & 1 \\
\hline 261 & 26 & 69 & 4 & 85 & 6 \\
\hline 265 & 23 & 67 & 1 & 69 & 5 \\
\hline 267 & 25 & 78 & 1 & 78 & 4 \\
\hline 274 & 27 & 82 & 10 & 82 & 3 \\
\hline 276 & 25 & 80 & 11 & 80 & 2 \\
\hline 277 & 22 & 58 & 28 & 58 & 1 \\
\hline 280 & 33 & 76 & 4 & 85 & 6 \\
\hline 284 & 30 & 74 & 1 & 69 & 5 \\
\hline 286 & 32 & 75 & 1 & 78 & 4 \\
\hline 293 & 34 & 76 & 10 & 82 & 3 \\
\hline 295 & 32 & 75 & 11 & 80 & 2 \\
\hline 296 & 29 & 75 & 28 & 58 & 1 \\
\hline 299 & 18 & 64 & 28 & 58 & 1 \\
\hline 303 & 21 & 64 & 11 & 80 & 2 \\
\hline 305 & 23 & 65 & 10 & 82 & 3 \\
\hline 312 & 21 & 64 & 1 & 78 & 4 \\
\hline 314 & 19 & 63 & 1 & 69 & 5 \\
\hline 315 & 22 & 65 & 4 & 85 & 6 \\
\hline 318 & 15 & 61 & 28 & 58 & 1 \\
\hline 322 & 18 & 61 & 11 & 80 & 2 \\
\hline 324 & 20 & 62 & 10 & 82 & 3 \\
\hline 331 & 18 & 61 & 1 & 78 & 4 \\
\hline 333 & 16 & 60 & 1 & 69 & 5 \\
\hline 334 & 19 & 62 & 4 & 85 & 6 \\
\hline
\end{tabular}


TABLE 3: Continued.

\begin{tabular}{|c|c|c|c|c|c|}
\hline Bus & Plug-in & $\begin{array}{c}\text { Plug- } \\
\text { out }\end{array}$ & $\begin{array}{l}\text { SOC } \\
\text { initial }\end{array}$ & $\begin{array}{c}\text { SOC } \\
\text { Req }\end{array}$ & Type \\
\hline 337 & 23 & 69 & 28 & 58 & 1 \\
\hline 341 & 26 & 69 & 11 & 80 & 2 \\
\hline 343 & 28 & 70 & 10 & 82 & 3 \\
\hline 350 & 26 & 69 & 1 & 78 & 4 \\
\hline 352 & 24 & 68 & 1 & 69 & 5 \\
\hline 353 & 27 & 70 & 4 & 85 & 6 \\
\hline 356 & 13 & 59 & 28 & 58 & 1 \\
\hline 360 & 16 & 59 & 11 & 80 & 2 \\
\hline 362 & 18 & 60 & 10 & 82 & 3 \\
\hline 369 & 16 & 59 & 1 & 78 & 4 \\
\hline 371 & 14 & 58 & 1 & 69 & 5 \\
\hline 372 & 17 & 60 & 4 & 85 & 6 \\
\hline 375 & 17 & 60 & 4 & 85 & 6 \\
\hline 379 & 14 & 58 & 1 & 69 & 5 \\
\hline 381 & 16 & 59 & 1 & 78 & 4 \\
\hline 388 & 18 & 60 & 10 & 82 & 3 \\
\hline 390 & 16 & 59 & 11 & 80 & 2 \\
\hline 391 & 13 & 59 & 28 & 58 & 1 \\
\hline 394 & 26 & 69 & 4 & 85 & 6 \\
\hline 398 & 23 & 67 & 1 & 69 & 5 \\
\hline 400 & 25 & 78 & 1 & 78 & 4 \\
\hline 407 & 27 & 82 & 10 & 82 & 3 \\
\hline 409 & 25 & 80 & 11 & 80 & 2 \\
\hline 410 & 22 & 58 & 28 & 58 & 1 \\
\hline 413 & 33 & 76 & 4 & 85 & 6 \\
\hline 417 & 30 & 74 & 1 & 69 & 5 \\
\hline 419 & 32 & 75 & 1 & 78 & 4 \\
\hline 426 & 34 & 76 & 10 & 82 & 3 \\
\hline 428 & 32 & 75 & 11 & 80 & 2 \\
\hline 429 & 29 & 75 & 28 & 58 & 1 \\
\hline 432 & 18 & 64 & 28 & 58 & 1 \\
\hline 436 & 21 & 64 & 11 & 80 & 2 \\
\hline 438 & 23 & 65 & 10 & 82 & 3 \\
\hline 445 & 21 & 64 & 1 & 78 & 4 \\
\hline 447 & 19 & 63 & 1 & 69 & 5 \\
\hline 448 & 22 & 65 & 4 & 85 & 6 \\
\hline
\end{tabular}

(v) The requested time for each CS must be greater than the minimum charging time required to charge the battery.

Since 22 variable filters will be placed in the network, the total unknown variables will become 44 , which are 22 locations and 22 optimal sizes. All these parameters will have their own constraints that need to be considered. In general, the parameters can be divided into 2 categories which are as follows:

(i) Filter location: the filter will be placed in low voltage bus to avoid any harmonic injection to upper feeder. There are 22 locations in 449 bus radial distribution system. Furthermore, only one VPF will be placed at

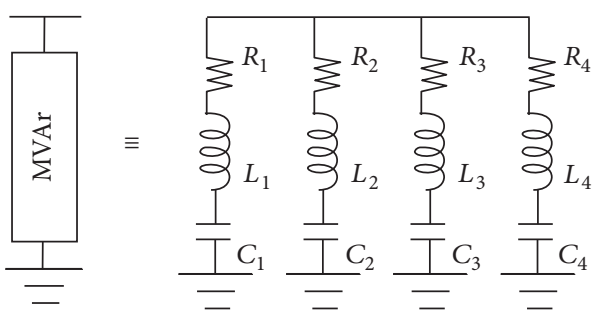

FIGURE 6: 1 set of filter.

every low voltage 415 buses system. The constraints are as follows:

$$
a_{i} \leq \text { Filter }_{i} \leq s_{i}, \quad i=1 \ldots 22 \text {. }
$$

(ii) Filter reactive value: each individual variable filter reactive value is limited to

$$
0 \mathrm{kVAr} \leq Q_{i} \leq 40 \mathrm{kVAr}, \quad i=1 \ldots 22
$$

2.6. Modelling of Passive Filter. The main function of a passive filter is to sink the harmonic current that flows in the system based on a selected frequency. The filter impedance will become very low to allow the harmonic to sink. Single tuned filter is the most popular type of filter which is used widely in dealing with harmonic pollution especially in the industrial area $[28,29]$. In this research, four units of single tuned filters are considered as one set of filter which can eliminate four frequencies as shown in Figure 6. Equation in [26] is used to calculate capacitor, inductance, and resistance components, respectively, as per (14). Capacitor is calculated based on injected reactive power $(Q)$ and voltage $(V)$ at that bus; meanwhile, inductance and resistance are based on the chosen harmonics $(n)$ that need to be reduced. Four sets of filter will be used to eliminate $3 \mathrm{rd}$, 5th, 7th, and 9th harmonic order in the network.

$$
\begin{aligned}
& C_{\text {Filter }}=\frac{Q}{2 \pi f V^{2}}, \\
& L_{\text {Filter }}=\frac{V^{2}}{2 n^{2} Q^{2} \pi f}, \\
& R_{\text {Filter }}=\frac{V^{2}}{n Q^{2}} .
\end{aligned}
$$

2.7. Multiobjective MLSA with Pareto Optimization. In this research, the optimization technique is used to identify optimized locations and sizes for 22 sets of filters in 449 bus radial distribution system. The Lightning Search Algorithm (LSA) is a metaheuristic optimization method inspired by lightning propagation from cloud to ground [30]. The LSA process basically starts with generation of random population before fitness calculation. The worst step leader will be eliminated every 10th iteration before the direction of the step leader is updated. Next, step leader movement is updated based on direction, shape parameter, and scale parameter. Lastly, forking phenomenon is taken place as per (7) for 
$1 \%$ of the better step leader. Since the problem faced in this research is too complicated with many local minima, modification on the existing LSA is needed. In this research, the MLSA is proposed with better convergence for this application compared to existing LSA. MLSA basically is the improvement of LSA method which is proven beneficial for this research.

The placement and sizing of variable filter will be based on five parameters, which are maximum $\mathrm{THD}_{V}$, maximum $\mathrm{THD}_{i}, \mathrm{THD}_{i}$ summation, average $\mathrm{THD}_{i}$, and apparent power losses for overall system. The three parameters that involve $\mathrm{THD}_{i}$ are to ensure that the $\mathrm{THD}_{i}$ at all buses is within acceptable range. These parameters are then normalized to get the most accurate solution as defined by (15)-(19). The weight summation with normalized fitness function is shown in (20) after several manual trials are done in order to get the best optimal result. Last but not least, basic equations to calculate $\mathrm{THD}_{i}$ and $\mathrm{THD}_{V}$ are as shown by (21) and (22).

$$
\begin{aligned}
\text { fit }_{1} & =\max \left(\mathrm{THD}_{V}\right), \quad n=1 \ldots 449, \\
\mathrm{fit}_{2} & =\max \left(\mathrm{THD}_{i}\right), \quad n=1 \ldots 30, \\
\mathrm{fit}_{3} & =\sum_{i=1}^{30} \mathrm{THD}_{i}, \\
\mathrm{fit}_{4} & =\frac{\sum_{i=1}^{30} \mathrm{THD}_{i}}{30}, \\
\text { fit }_{5} & =\sum_{i=1}^{449} s_{i-\text { loss }}, \\
\text { Fit } & =0.1 \mathrm{fit}_{1}+0.2 \mathrm{fit}_{2}+0.2 \mathrm{fit}_{3}+0.2 \mathrm{fit}_{4}+0.3 \mathrm{fit}_{5}, \\
\mathrm{THD}_{V} & =\frac{\sqrt{\sum_{h=2}^{9} V_{h}^{2}}}{V_{1}} \times 100 \%, \\
\mathrm{THD}_{i} & =\frac{\sqrt{\sum_{h=2}^{9} I_{h}^{2}}}{I_{1}} \times 100 \% .
\end{aligned}
$$

In order to reduce computational time in this research, bus location is gathered from MLSA with weight summation approach; meanwhile, Pareto optimization is used to determine size of VPF. Normally, Pareto technique will produce a "nondominated" solution which has more than a single solution [31]. Thus, fuzzy satisfying approach is adopted in this research to get the best solution from the "nondominated" solutions. Equation (23) is the weight membership function used in fuzzy approach for all "nondominated" solutions in the fuzzy set. The fit ${ }_{\max }$ in the formula represents the existing fitness value before implementing the filters, while $m$ represents the number of solutions listed in the "nondominated" list. The highest $\mu_{j}$ value indicates the best solution for this research.

$$
\mu_{\text {fit }_{-j}}=\left\{\begin{array}{lc}
1, & \text { fit }_{j} \leq 0 \\
\text { fit }_{\text {max }}-\text { fit }_{j} & 0<\text { fit }_{j}<\text { fit }_{\text {max }} \\
0, & \text { fit }_{j} \geq \text { fit }_{\text {max }},
\end{array}\right.
$$

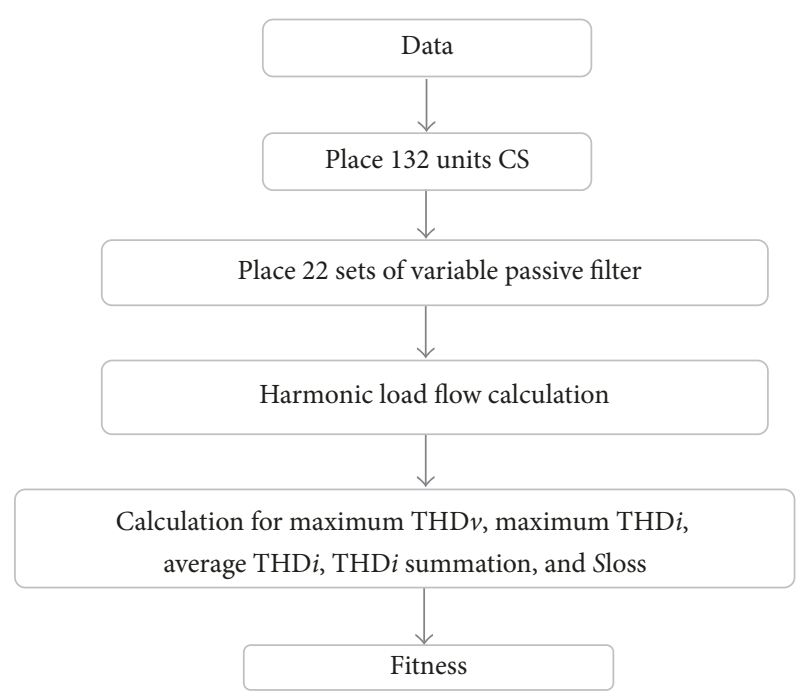

FIGURE 7: Flowchart to evaluate fitness.

$$
\mu_{j}=\frac{\sum_{1}^{5} \mu_{\text {fit }-j}}{\sum_{1}^{m} \sum_{1}^{5} \mu_{\text {fit }-j}} .
$$

\section{Methodology}

The variable filter is used to minimize the impact of harmonics due to large-scale deployment of CS in the big distribution system. The numbers of filter are based on how severe the harmonic distortion and power losses on the existing system. By placing 132 CS units (6 units for every low voltage residential area) at specific locations, MLSA will be used to determine optimal placement and size for 22 sets of filters (1 set for every low voltage residential area). The $\mathrm{THD}_{V}$, $\mathrm{THD}_{i}$, and $S_{\text {loss }}$ will be recorded based on specify CS and filter locations. The overall process to evaluate system performance is as shown in Figure 7.

There are four modifications on MLSA that are made to improve LSA method. The first modification is on channel time; the original channel time is not suitable when dealing with the problems that have many local minima. The second modification is the updating approach; a new updating approach able to check the forward or backward direction is proposed. The third modification is on the scale parameter; in LSA, it is based on exponential distribution, which will cause the step leader movement to be active at $20 \%$ of the early iterations as per (24). Thus, in MLSA, the Laplacian distribution equation is used to increase the movement of the step leader during 30\% until $70 \%$ iterations. Last but not least, the learning factors which make the movement of the step leader more active is implemented in the fourth modification as per (25).

$$
\begin{aligned}
P_{i \_ \text {new }}^{L} & =P^{L}{ }_{i} \pm \text { normrand }\left(\mu_{i}, \sigma_{i}\right), \\
\mu_{i} & =R\left(x_{i}-x_{\text {best }}\right),
\end{aligned}
$$




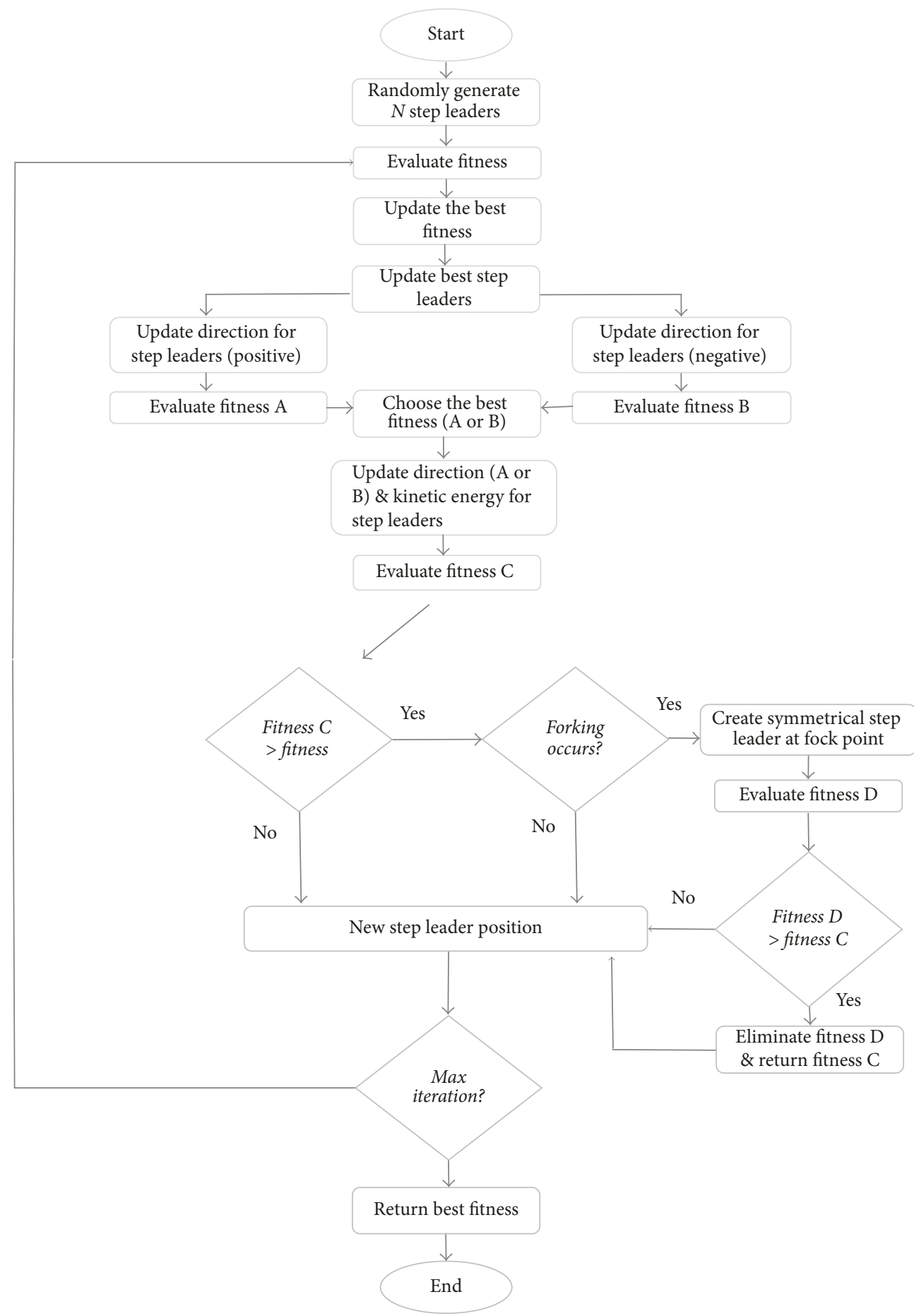

FIGURE 8: Flowchart for MLSA method to get minimum fitness with weightage summation approach.

where $\sigma_{i}$ is the scale parameter at $i$ th iteration $(1 / 3) e^{-(t-50 / 15)}$, $R$ is learning factor (2.0 in this research), $P^{L}{ }_{i \text { new }}$ is new position in lead projectile at $i$ th iteration, $P^{L}{ }_{i}$ is position in lead projectile at $i$ th iteration, $\mu_{i}$ is shape parameter at $i$ th iteration, $x_{i}$ is the position at $i$ th iteration, and $x_{\text {best }}$ is the best individual during minimum.

Figure 8 shows the flowchart for MLSA optimization process using weightage summation approach for this research.
In this process, simulation involves 500 iterations and 50 populations. Next, the bus location results will be used in the next stage by the MLSA-Pareto-Fuzzy combination technique. Figure 9 shows the flowchart for MLSA optimization process with Pareto-Fuzzy technique. In this stage, Pareto technique will be adopted in finding the best solution, while fuzzy approach will be used at the end of process to find the best solution among nondominated solutions. 


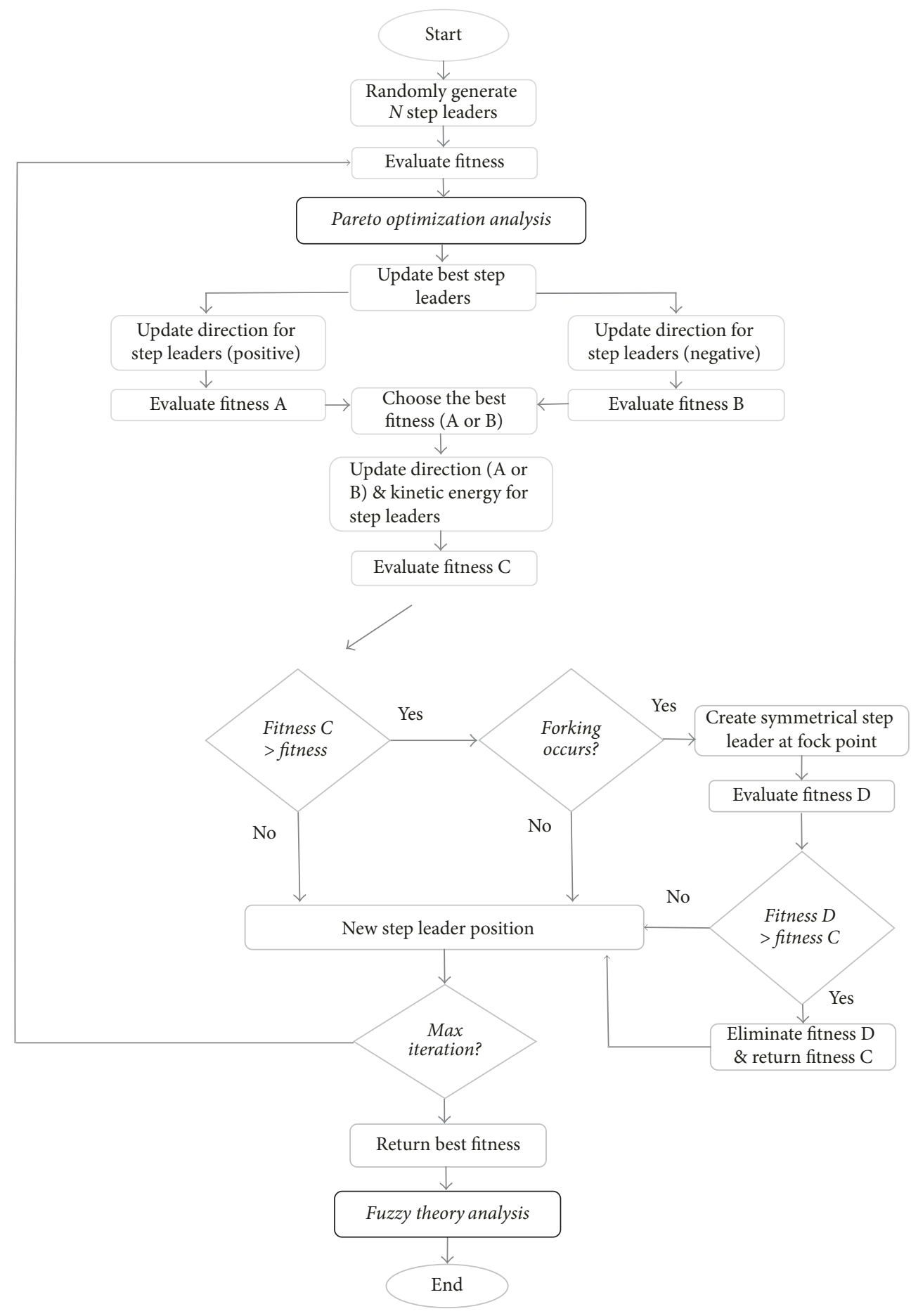

FIGURE 9: Flowchart for MLSA method to get minimum fitness with Pareto-Fuzzy technique.

\section{Result and Discussion}

The simulation on this research was done based on the scenario where $132 \mathrm{CS}$ units are installed in low voltage $415 \mathrm{~V}$ buses for 24 hours (96 states, 1 state $=15$ minutes). The schedule for CS operation can be referred to in Table 3. The $\mathrm{THD}_{V}$ and $\mathrm{THD}_{i}$ for all medium voltage buses and lines are shown in Figures 11 and 12, respectively, for sampling time at 30 and 63 . Sampling time 63 is chosen due to the worst harmonic recorded in 24 hours, while sampling time 30 is chosen due to the lowest harmonic injection. From the result, without VPF, maximum $\mathrm{THD}_{V}$ for sampling time 30 is recorded at bus 15 with $0.8750 \%$ and maximum $\mathrm{THD}_{i}$ is recorded at lines between buses 26 and 27 (line number 27 ) with the value $0.6780 \%$. For sampling time 63 , maximum $\mathrm{THD}_{V}$ is $0.9032 \%$ at bus 15 , while maximum $\mathrm{THD}_{i}$ 
TABLE 4: Bus location and filter size after 500 iterations.

\begin{tabular}{lcc}
\hline Number & Bus location & Filter size (kVAr) \\
\hline$(1)$ & 42 & 28.007 \\
$(2)$ & 56 & 16.494 \\
$(3)$ & 85 & 22.724 \\
$(4)$ & 105 & 1.018 \\
$(5)$ & 119 & 1.777 \\
$(6)$ & 145 & 24.54 \\
$(7)$ & 161 & 28.731 \\
$(8)$ & 168 & 27.679 \\
$(9)$ & 193 & 29.999 \\
$(10)$ & 217 & 8.927 \\
$(11)$ & 222 & 2.109 \\
$(12)$ & 254 & 1.024 \\
$(13)$ & 275 & 27.391 \\
$(14)$ & 282 & 17.989 \\
$(15)$ & 305 & 14.646 \\
$(16)$ & 322 & 24.733 \\
$(17)$ & 337 & 26.043 \\
$(18)$ & 367 & 24.758 \\
$(19)$ & 387 & 29.863 \\
$(20)$ & 407 & 21.946 \\
$(21)$ & 424 & 26.069 \\
$(22)$ & 447 & 28.156 \\
\hline
\end{tabular}

TABLE 5: Result using MLSA with multiobjective function.

\begin{tabular}{lcc}
\hline Function & Existing & After multiobjective function \\
\hline Max $\mathrm{THD}_{V}$ & 1.258004 & 0.74478 \\
$\mathrm{Max} \mathrm{THD}_{i}$ & 2.019816 & 1.16256 \\
$\mathrm{THD}_{i}$ average & 0.474635 & 0.322456 \\
$\mathrm{THD}_{i}$ sum & 14.71369 & 9.996144 \\
$S_{\text {loss }}$ & 0.04175 & 0.043149 \\
Fitness & 1 & 0.75612 \\
\hline
\end{tabular}

is recorded at lines between buses 12 and 13 (line number 17) with the value $1.4166 \%$. Apparent losses for both sampling times are $90.3 \mathrm{kVA}$ and $40.4 \mathrm{kVA}$, respectively.

Next, 22 sets of VPFs are placed at low voltage $415 \mathrm{~V}$ buses using MLSA optimization with multiobjective function. The optimal location and size for all filters are tabulated in Table 4, while the parameter value is shown at Table 5 . From the results in Table 5, there is a significant reduction for maximum $\mathrm{THD}_{V}$ from $1.258004 \%$ to $0.74478 \%$ and maximum $\mathrm{THD}_{i}$ from $2.019816 \%$ to $1.16256 \%$ after MLSA get the optimal placement for VPF. However, the total apparent losses in the network are increased. Thus, a Pareto-Fuzzy approach is used to cater this issue at the next step.

The results from Pareto consist of multiple set solutions where the possible solution is the best solution as long as one of their objectives functions is dominant compared to other solutions. Fuzzy approach will be used to find the best among solutions in Pareto set. In this study, the solution consists of $\mathrm{THD}_{V}, \mathrm{THD}_{i}$ (minimum, average, and summation), and power loss. From the analysis, the total Pareto result for

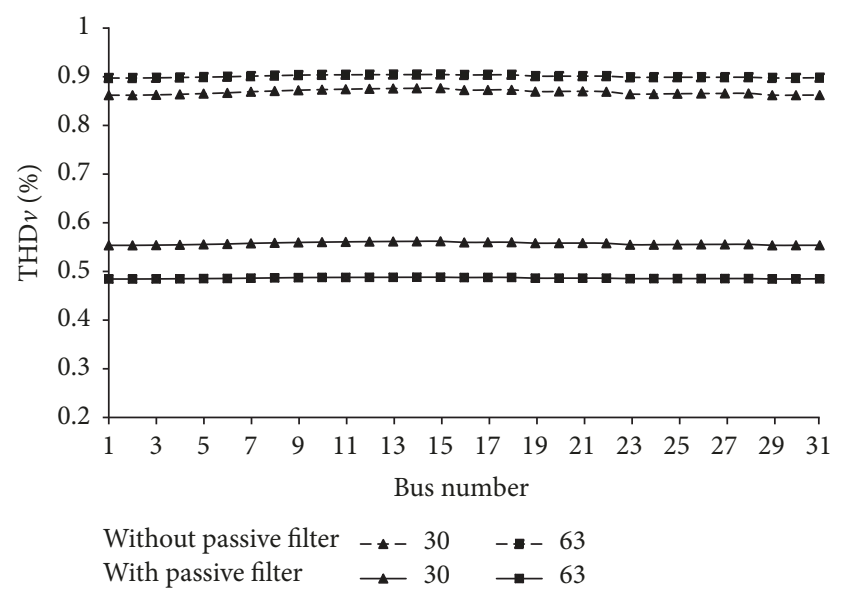

FIgURE 10: $\mathrm{THD}_{V}$ at medium voltage for times 30 and 63 with and without passive filter.

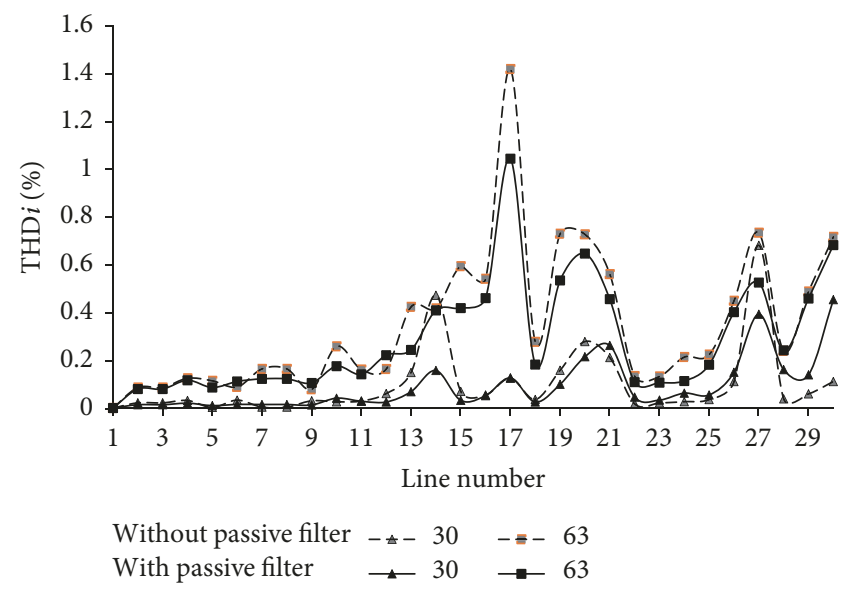

FIgURE 11: $\mathrm{THD}_{i}$ at medium voltage for times 30 and 63 with and without passive filter.

sampling time at 63 in MLSA is 137 sets. The fuzzy approach is used in order to get the best single solution. The highest value of weight membership function in fuzzy approach will be chosen as the best solution in this research. Tables 6 and 7 show the five best solutions with the highest $\mu_{j}$ for sampling times 63 and 30. From the result, the best sizing for all filters is shown in Table 8 at sampling time 63. The size of filter to cater harmonic is different between times 30 and 63 which indicates that the size of passive filter is very crucial in this study. The $\mathrm{THD}_{V}$ and $\mathrm{THD}_{i}$ for buses and lines, after the filter implementation as per MLSA with Pareto-Fuzzy best solution, are shown in Figures 10 and 11. From the results, it shows significant reduction of $\mathrm{THD}_{V}$ at all medium voltage buses, while majority of $\mathrm{THD}_{i}$ also shows reduction. From the best solution, the $\mathrm{THD}_{i}$ and $\mathrm{THD}_{v}$ for the 449 bus distribution system have been reduced effectively after filter installations. The results for all 96 slots are shown in Figures 12-16. Based on Figures 12 and 13, maximum $\mathrm{THD}_{V}$ and $\mathrm{THD}_{i}$ show reduction for all states which indicate that the system is better compared to previous 
TABLE 6: The five best solutions based on the highest $\mu_{j}$ using fuzzy approach for 137 sets of solutions (sampling time $=63$ ).

\begin{tabular}{|c|c|c|c|c|c|c|c|c|c|c|c|}
\hline \multirow{2}{*}{ Number } & \multicolumn{5}{|c|}{ Fitness parameter } & \multirow{2}{*}{$\mu_{\text {fit }}^{1}$} & \multirow{2}{*}{$\mu_{\mathrm{ftt}}^{2}$} & \multirow{2}{*}{$\mu_{\mathrm{fit}}^{3}$} & \multirow{2}{*}{$\mu_{\mathrm{fit}}^{4}$} & \multirow{2}{*}{$\mu_{\mathrm{fit}}^{5}$} & \multirow[b]{2}{*}{$\mu_{j}$} \\
\hline & $\mathrm{fit}_{1}$ & $\mathrm{fit}_{2}$ & $\mathrm{fit}_{3}$ & $\mathrm{fit}_{4}$ & $\mathrm{fit}_{5}$ & & & & & & \\
\hline (1) & 0.731905 & 1.042406 & 0.308344 & 9.558658 & 0.040295 & 0.350982 & 0.26416 & 0.196401 & 0.196401 & 0.004568 & 0.009541 \\
\hline (2) & 0.715371 & 1.087904 & 0.308852 & 9.574414 & 0.040469 & 0.365644 & 0.232043 & 0.195076 & 0.195076 & 0.000013 & 0.009308 \\
\hline (3) & 0.691356 & 1.213682 & 0.299624 & 9.288355 & 0.040021 & 0.386939 & 0.143255 & 0.219125 & 0.219125 & 0.011102 & 0.00923 \\
\hline (4) & 0.691356 & 1.213682 & 0.299624 & 9.288355 & 0.040021 & 0.386939 & 0.143255 & 0.219125 & 0.219125 & 0.011102 & 0.00923 \\
\hline (5) & 0.691356 & 1.213682 & 0.299624 & 9.288355 & 0.040021 & 0.386939 & 0.143255 & 0.219125 & 0.219125 & 0.011102 & 0.00923 \\
\hline
\end{tabular}

TABLE 7: The five best solutions based on the highest $\mu_{j}$ using fuzzy approach for 10 sets of solutions (sampling time $=30$ ).

\begin{tabular}{|c|c|c|c|c|c|c|c|c|c|c|c|}
\hline \multirow{2}{*}{ Number } & \multicolumn{5}{|c|}{ Fitness parameter } & \multirow{2}{*}{$\mu_{\text {fit }}^{1}$} & \multirow{2}{*}{$\mu_{\text {fit }}^{2}$} & \multirow{2}{*}{$\mu_{\text {fit }}^{3}$} & \multirow{2}{*}{$\mu_{\text {fit }}^{4}$} & \multirow{2}{*}{$\mu_{\text {fit }}^{5}$} & \multirow{2}{*}{$\mu_{j}$} \\
\hline & $\mathrm{fit}_{1}$ & fit $_{2}$ & $\mathrm{fit}_{3}$ & $\mathrm{fit}_{4}$ & $\mathrm{fit}_{5}$ & & & & & & \\
\hline (1) & 0.62029 & 0.452838 & 0.093176 & 2.888464 & 0.089405 & 0.34565 & 0.334048 & 0.081789 & 0.081789 & 0.009764 & 0.124558 \\
\hline (2) & 0.647588 & 0.41204 & 0.095228 & 2.952076 & 0.089555 & 0.316852 & 0.394046 & 0.061568 & 0.061568 & 0.0081 & 0.122965 \\
\hline (3) & 0.639053 & 0.421681 & 0.097018 & 3.007568 & 0.089525 & 0.325856 & 0.379868 & 0.043927 & 0.043927 & 0.00843 & 0.117106 \\
\hline (4) & 0.639053 & 0.421681 & 0.097018 & 3.007568 & 0.089525 & 0.325856 & 0.379868 & 0.043927 & 0.043927 & 0.00843 & 0.117106 \\
\hline (5) & 0.652162 & 0.410311 & 0.10102 & 3.131623 & 0.089686 & 0.312028 & 0.396589 & 0.004492 & 0.004492 & 0.006651 & 0.105752 \\
\hline
\end{tabular}

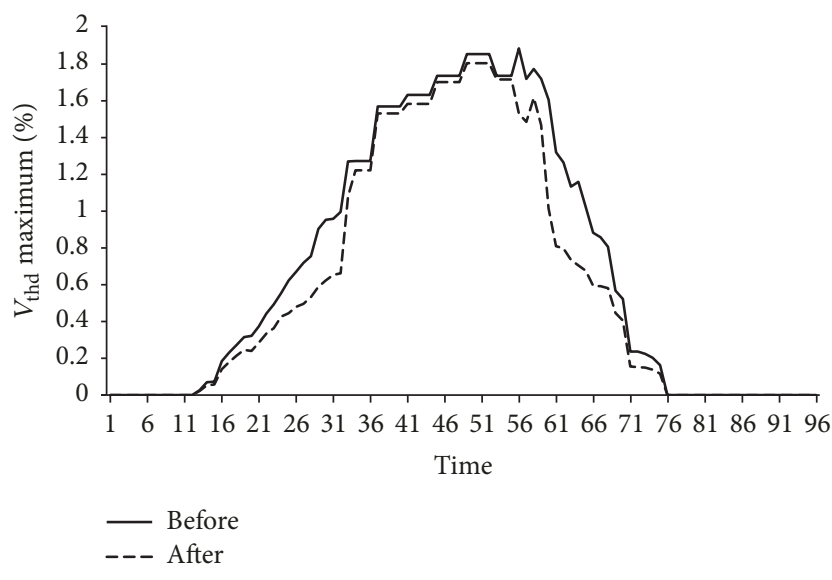

FIGURE 12: $\mathrm{THD}_{v}$ maximum for 24 hours with and without passive filter.

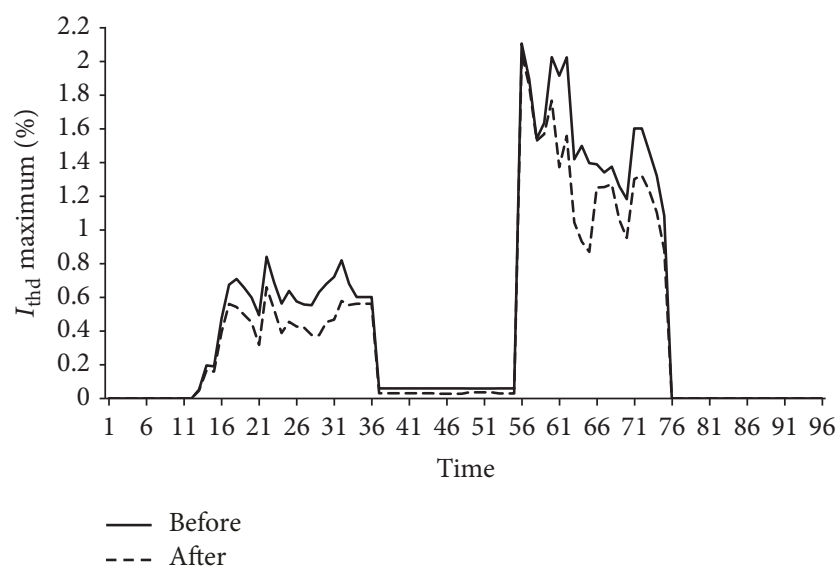

FIgURE 13: $\mathrm{THD}_{i}$ maximum for 24 hours with and without passive filter.

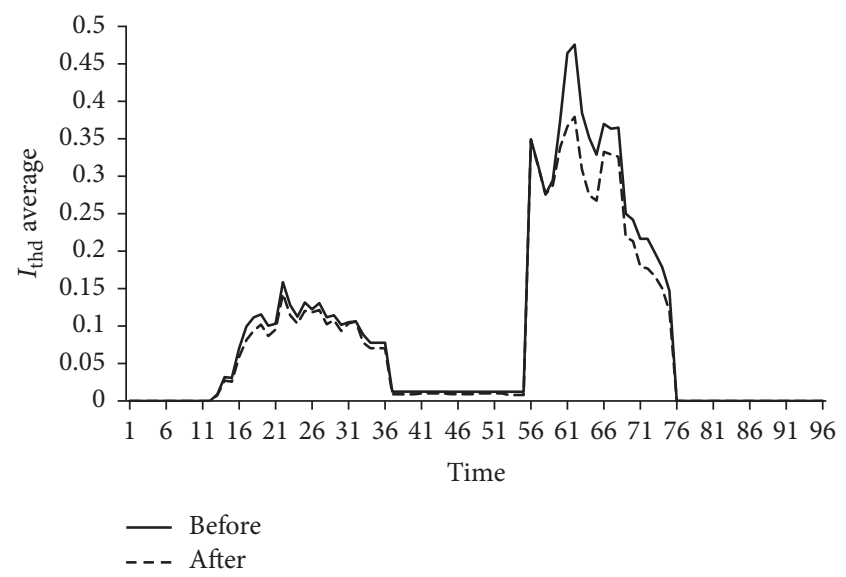

FIGURE 14: $\mathrm{THD}_{i}$ average for 24 hours with and without passive filter.

value, while apparent losses at Figure 16 show the capability of this method to also reduce losses together with harmonic. Next, Figures 14 and 15 also show reduction which assist $\mathrm{THD}_{i}$ improvement in overall system indirectly. From the result, implementation of VPF with changes every 15 minutes is able to reduce harmonic for 24 hours. Other than that, it is obvious that the implementation of MLSA with Pareto-Fuzzy is able to identify the best solution that provides the greatest improvement to the overall system.

Based on the collective result gathered from all 96 simulations that represent 24 hours, minimum and maximum values of VPF at dedicated bus are acquired. Table 9 shows the minimum and maximum $V P F$ required to solve harmonic problem after EV is installed.

\section{Conclusion}

Optimal placement and sizing of VPF in the distribution system can avoid harmonic injection to the medium voltage 
TABLE 8: Filter size based on the best solution after fuzzy at sampling times 30 and 63.

\begin{tabular}{lccc}
\hline \multirow{2}{*}{ Number } & Bus location & \multicolumn{2}{c}{ Filter size (MVAr) } \\
& & $($ Time $=30)$ & $($ Time $=63)$ \\
\hline$(1)$ & 42 & 0.017278 & 0.025038 \\
$(2)$ & 56 & 0.02187 & 0.005959 \\
$(3)$ & 85 & 0.010198 & 0.019481 \\
$(4)$ & 105 & 0.029332 & 0.011044 \\
$(5)$ & 119 & 0.014816 & 0.001792 \\
$(6)$ & 145 & 0.019336 & 0.02311 \\
$(7)$ & 161 & 0.002469 & 0.026056 \\
$(8)$ & 168 & 0.023573 & 0.022019 \\
$(9)$ & 193 & 0.003353 & 0.018806 \\
$(10)$ & 217 & 0.018943 & 0.008713 \\
$(11)$ & 222 & 0.019898 & 0.016408 \\
$(12)$ & 254 & 0.024081 & 0.002977 \\
$(13)$ & 275 & 0.0176 & 0.018999 \\
$(14)$ & 282 & 0.020249 & 0.029898 \\
$(15)$ & 305 & 0.01956 & 0.022548 \\
$(16)$ & 322 & 0.016437 & 0.015687 \\
$(17)$ & 337 & 0.014686 & 0.01437 \\
$(18)$ & 367 & 0.026797 & 0.016679 \\
$(19)$ & 387 & 0.022292 & 0.012124 \\
$(20)$ & 407 & 0.011888 & 0.016332 \\
$(21)$ & 424 & 0.001049 & 0.011097 \\
$(22)$ & 447 & 0.020735 & 0.028984 \\
\hline & & &
\end{tabular}

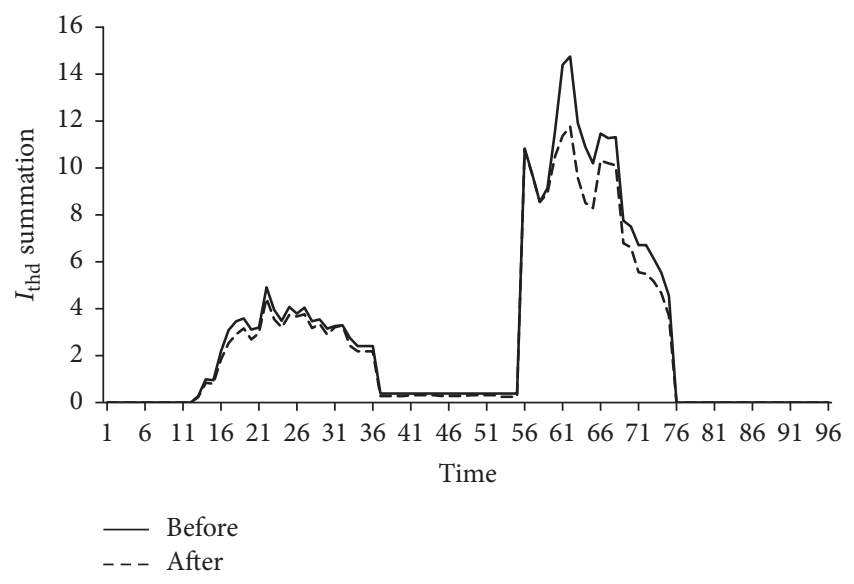

FIGURE 15: $\mathrm{THD}_{i}$ summation for 24 hours with and without passive filter.

network as well as reducing apparent power losses. This research has shown that MLSA with multiobjective function and Pareto-Fuzzy are able to determine appropriate filter location and size to reduce harmonic distortion and apparent losses in 449 bus system with 132 units of CS. Furthermore, the placement of twenty-two sets of four unit single-tuned filters is able to reduce the four harmonic orders. Based on simulation run for every 15 minutes, the proposed technique is able to improve maximum $\mathrm{THD}_{V}$, maximum $\mathrm{THD}_{i}$, and $S_{\text {loss }}$ up to $39.14 \%, 52.5 \%$, and $2.96 \%$, respectively. The study
TABLE 9: Minimum and maximum VPF size based on 96 simulations.

\begin{tabular}{lccc}
\hline \multirow{2}{*}{ Number } & Bus location & \multicolumn{2}{c}{ Filter size (kVAr) } \\
& & Minimum & Maximum \\
\hline$(1)$ & 42 & 1.021 & 28.916 \\
$(2)$ & 56 & 1.282 & 28.659 \\
$(3)$ & 85 & 0.619 & 28.838 \\
$(4)$ & 105 & 0.637 & 29.498 \\
$(5)$ & 119 & 0.692 & 20.457 \\
$(6)$ & 145 & 0.855 & 29.559 \\
$(7)$ & 161 & 0.204 & 29.944 \\
$(8)$ & 168 & 0.5 & 29.769 \\
$(9)$ & 193 & 0.725 & 18.806 \\
$(10)$ & 217 & 0.724 & 29.936 \\
$(11)$ & 222 & 0.228 & 29.927 \\
$(12)$ & 254 & 0.319 & 29.996 \\
$(13)$ & 275 & 0.171 & 28.969 \\
$(14)$ & 282 & 0.409 & 29.898 \\
$(15)$ & 305 & 0.654 & 29.805 \\
$(16)$ & 322 & 0.318 & 29.859 \\
$(17)$ & 337 & 0.84 & 29.565 \\
$(18)$ & 367 & 0.176 & 29.56 \\
$(19)$ & 387 & 1.385 & 29.576 \\
$(20)$ & 407 & 0.235 & 26.327 \\
$(21)$ & 424 & 1.049 & 28.326 \\
$(22)$ & 447 & 0.244 & 29.922 \\
\hline & & &
\end{tabular}

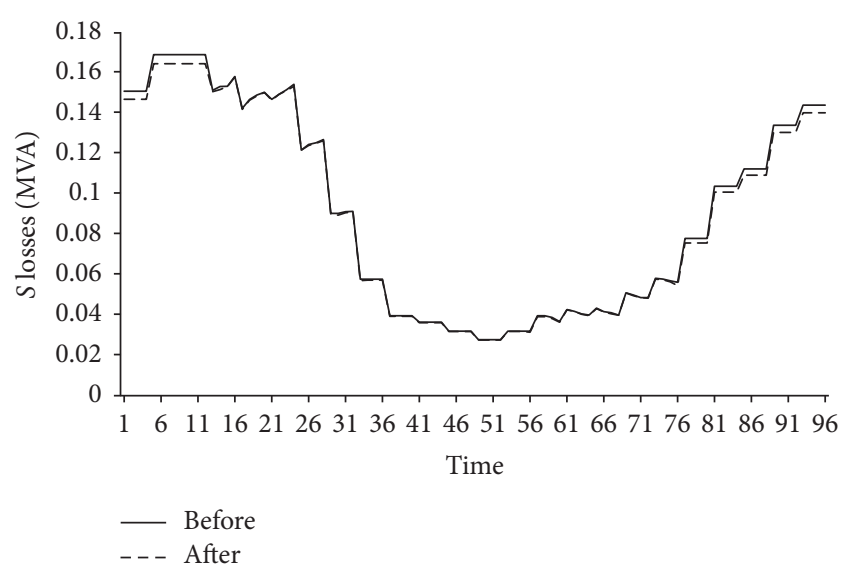

FIgURE 16: Apparent power losses for 24 hours with and without passive filter.

is very important for future distribution grid that might have many CS in the network. For future work, the study can be expanded to other types of passive filter able to have better impact on the system such as C-type filter and others.

\section{Conflicts of Interest}

The authors declare that they have no conflicts of interest. 


\section{Acknowledgments}

The researchers would like to express their appreciation to the Universiti Teknologi Malaysia (UTM) for supporting this work through GUP Grant (15H89).

\section{References}

[1] World Balance, https://www.iea.org/Sankey/\#?c=World\&s=Finalconsumption.

[2] A. Lucas, F. Bonavitacola, E. Kotsakis, and G. Fulli, "Grid harmonic impact of multiple electric vehicle fast charging," Electric Power Systems Research, vol. 127, pp. 13-21, 2015.

[3] J. Niitsoo, J. Kilter, I. Palu, P. Taklaja, and L. Kütt, "Harmonic levels of domestic and electrical vehicle loads in residential distribution networks," in Proceedings of the IEEE (AFRICON '13), pp. 184-188, Mauritius, September 2013.

[4] S. Pazouki, A. Mohsenzadeh, M.-R. Haghifam, and S. Ardalan, "Simultaneous allocation of charging stations and capacitors in distribution networks improving voltage and power loss," Canadian Journal of Electrical and Computer Engineering, vol. 38, no. 2, Article ID 7097124, pp. 100-105, 2015.

[5] M. S. Taci, M. H. Sarul, and G. Yildirmaz, "Effects of the harmonic components upon transformer active losses in case of (non) sinusoidal sources and (non) linear loads," in Proceedings of the IEEE International Conference on Industrial Technology 2000, vol. 1, pp. 741-746, IEEE, January 2000.

[6] N. Zhou, J. Wang, Q. Wang, N. Wei, and X. Lou, "Capacity calculation of shunt active power filters for electric vehicle charging stations based on harmonic parameter estimation and analytical modeling," Energies, vol. 7, no. 8, pp. 5425-5443, 2014.

[7] M. Alonso, H. Amaris, J. G. Germain, and J. M. Galan, "Optimal charging scheduling of electric vehicles in smart grids by heuristic algorithms," Energies, vol. 7, no. 4, pp. 2449-2475, 2014.

[8] G. R. Bharati and S. Paudyal, "Coordinated control of distribution grid and electric vehicle loads," Electric Power Systems Research, vol. 140, pp. 761-768, 2016.

[9] M. A. S. Masoum and S. M. H. Nabavi, "Hybrid optimal onlineovernight charging coordination of plug-in electric vehicles in smart grid," Journal of Power Sources, vol. 330, pp. 7-17, 2016.

[10] B. P. De Campos, L. A. R. De Sousa, and P. F. Ribeiro, "Mitigation of harmonic distortion with passive filters," in Proceedings of the 17th International Conference on Harmonics and Quality of Power, (ICHQP '16), pp. 646-651, IEEE, Brazil, October 2016.

[11] K. H. Shafad, J. J. Jamian, and S. A. S. Nasir, "Harmonic distortion mitigation for multiple modes charging station via optimum passive filter design," in Proceedings of the 2016 IEEE Conference on Systems, Process and Control, (ICSPC '16), pp. 219-223, Malaysia, December 2016.

[12] V.-L. Nguyen, T. Tran-Quoc, and S. Bacha, "Harmonic distortion mitigation for electric vehicle fast charging systems," in Proceedings of the 2013 IEEE Grenoble Conference PowerTech, (POWERTECH '13), pp. 1-6, IEEE, Grenoble, France, June 2013.

[13] C. C. Hao, Y. J. Tang, and J. Shi, "Study on the harmonic impact of large scale electric vehicles to grid," AMM Applied Mechanics and Materials, vol. 443, pp. 273-278, 2013.

[14] S. Pirouzi, M. A. Latify, and G. R. Yousefi, "Investigation on reactive power support capability of PEVS in distribution network operation," in Proceedings of the 23rd Iranian Conference on Electrical Engineering, (ICEE '15), pp. 1591-1596, IEEE, Tehran, Iran, May 2015.
[15] Y. Cho and H. Cha, "Single-tuned passive harmonic filter design considering variances of tuning and quality factor," Journal of International Council on Electrical Engineering, vol. 1, pp. 7-13, 2011.

[16] S. Sakar, M. E. Balci, S. H. E. Abdel Aleem, and A. F. Zobaa, "Increasing PV hosting capacity in distorted distribution systems using passive harmonic filtering," Electric Power Systems Research, vol. 148, pp. 74-86, 2017.

[17] S. Farkoush, C. Kim, and S. Rhee, "THD reduction of distribution system based on ASRFC and HVC method for SVC under EV charger condition for power factor improvement," Symmetry, vol. 8, no. 12, p. 156, 2016.

[18] G. Chang, S. Chu, and H. Wang, "A simplified forward and backward sweep approach for distribution system load flow analysis," in Proceedings of the International Conference on Power System Technology, pp. 1-5, 2006.

[19] M. Abdel-Akher, "Voltage stability analysis of unbalanced distribution systems using backward/forward sweep load-flow analysis method with secant predictor," IET Generation, Transmission \& Distribution, vol. 7, no. 3, pp. 309-317, 2013.

[20] P. Samal and S. Ganguly, "A modified forward backward sweep load flow algorithm for unbalanced radial distribution systems," in Proceedings of the IEEE Power and Energy Society General Meeting, (PESGM '15), pp. 1-5, IEEE, Denver, CO, USA, July 2015.

[21] C. S. Gupta, "Review of forward \& backward sweep method for load flow analysis of radial distribution system," IJAREEIE International Journal of Advanced Research in Electrical, Electronics and Instrumentation Engineering, vol. 04, pp. 5595-5599, 2015.

[22] R. Bass and N. Zimmerman, "Impacts of electric vehicle charging on electric power distribution systems," OTREC-SS-731. Portland, OR: Transportation Research and Education Center (TREC), 2013.

[23] S. A. Kumar and K. R. Reddy, "Computation of the power flow solution of a radial distribution system for harmonic components," International Journal of Energy, 2013.

[24] J.-H. Teng, S.-H. Liao, and R.-C. Leou, “Three-phase harmonic analysis method for unbalanced distribution systems," Energies, vol. 7, no. 1, pp. 365-384, 2014.

[25] J.-H. Teng and C.-Y. Chang, "Backward/forward sweep-based harmonic analysis method for distribution systems," IEEE Transactions on Power Delivery, vol. 22, no. 3, pp. 1665-1672, 2007.

[26] S. M. Halpin, P. F. Ribeiro, and J. J. Dai, "Frequency-domain harmonic analysis methods," IEEE Power Engineering Society: Tutorial in Harmonics Modeling and Simulation, pp. 49-54, 1998.

[27] C. Zhang, J. Jiang, L. Zhang, S. Liu, L. Wang, and P. C. Loh, "A generalized SOC-OCV model for lithium-ion batteries and the SOC estimation for LNMCO battery," Energies, vol. 9, no. 11, article no. 900, 2016.

[28] M. Awadalla, M. Omer, and A. Mohamed, "Single-tuned filter design for harmonic mitigation and optimization with capacitor banks," in Proceedings of the 1st International Conference on Computing, Control, Networking, Electronics and Embedded Systems Engineering, (ICCNEEE '15), pp. 242-247, IEEE, Khartoum, Sudan, September 2015.

[29] D. M. Soomro and M. M. Almelian, "Optimal design of a single tuned passive filter to mitigate harmonics in power frequency," ARPN Journal of Engineering and Applied Sciences, vol. 10, no. 19, pp. 9009-9014, 2015. 
[30] H. Shareef, A. A. Ibrahim, and A. H. Mutlag, "Lightning search algorithm," Applied Soft Computing, vol. 36, pp. 315-333, 2015.

[31] R. N. S. R. Mukhtaruddin, H. A. Rahman, M. Y. Hassan, and J. J. Jamian, "Optimal hybrid renewable energy design in autonomous system using Iterative-Pareto-Fuzzy technique," International Journal of Electrical Power \& Energy Systems, vol. 64, pp. 242-249, 2015. 


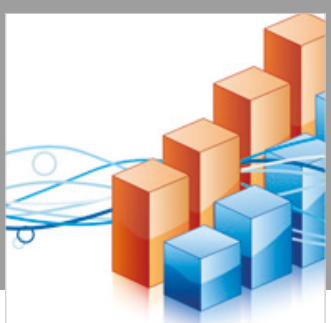

Advances in

Operations Research

\section{-n-m}
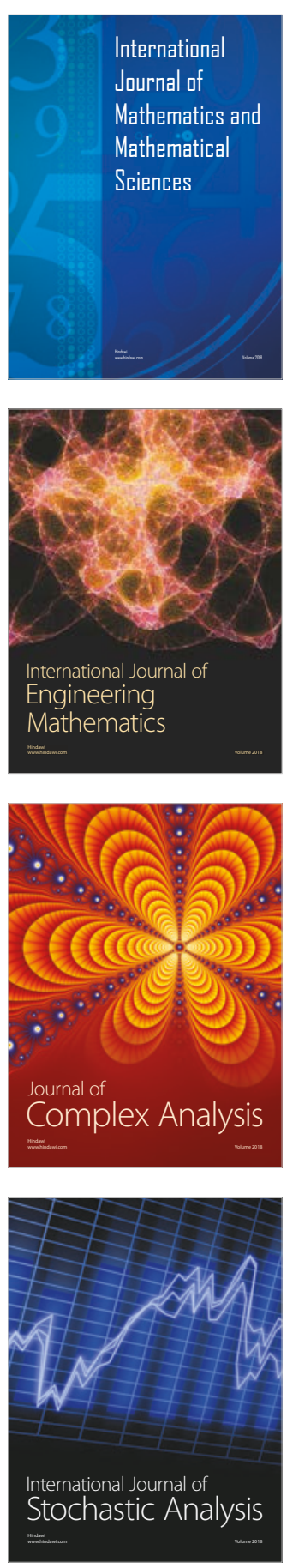
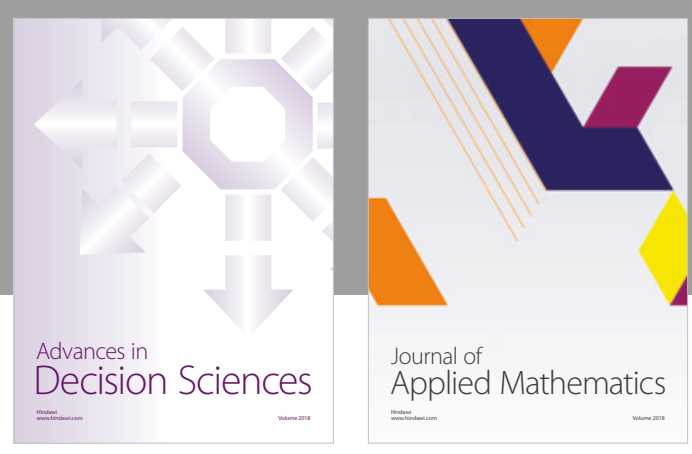

Journal of

Applied Mathematics
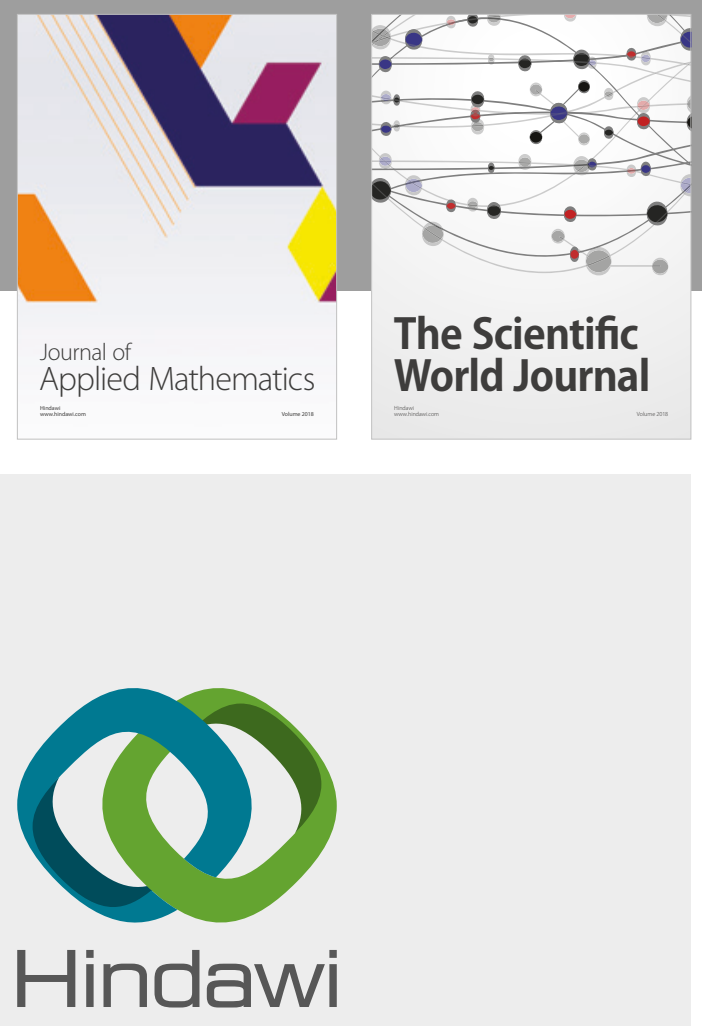

Submit your manuscripts at

www.hindawi.com

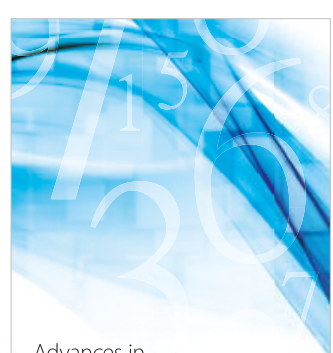

Advances in
Numerical Analysis
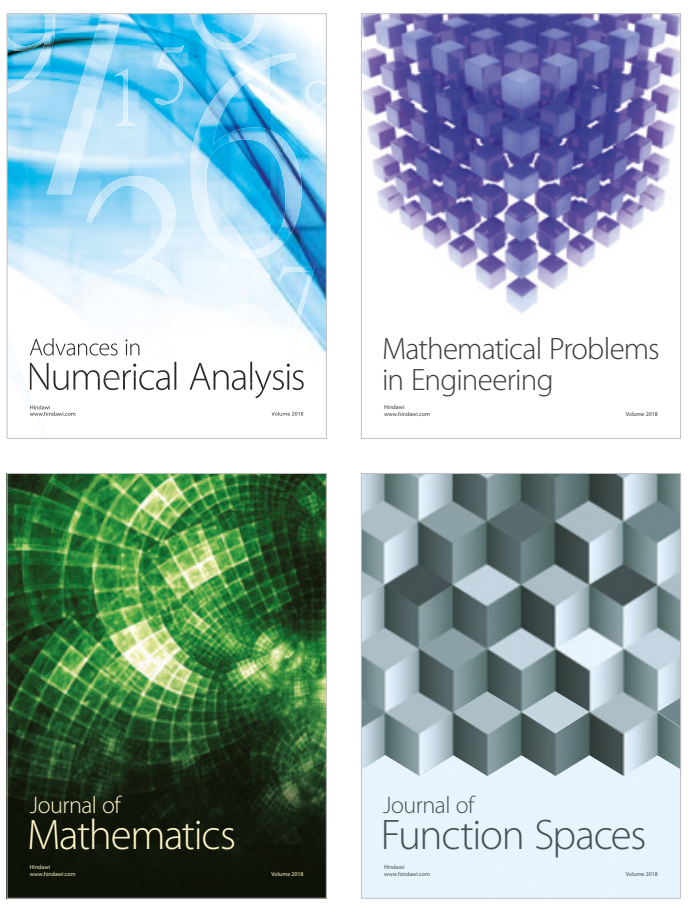

Mathematical Problems in Engineering

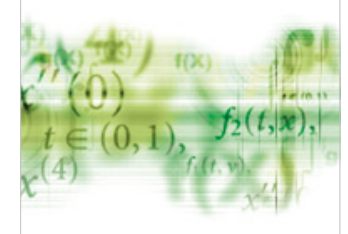

International Journal of

Differential Equations

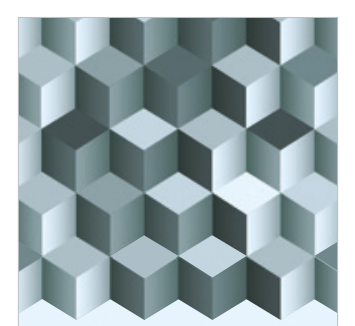

Journal of

Function Spaces
The Scientific

World Journal

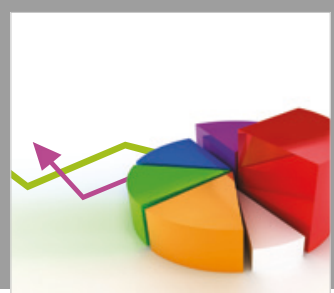

Journal of

Probability and Statistics
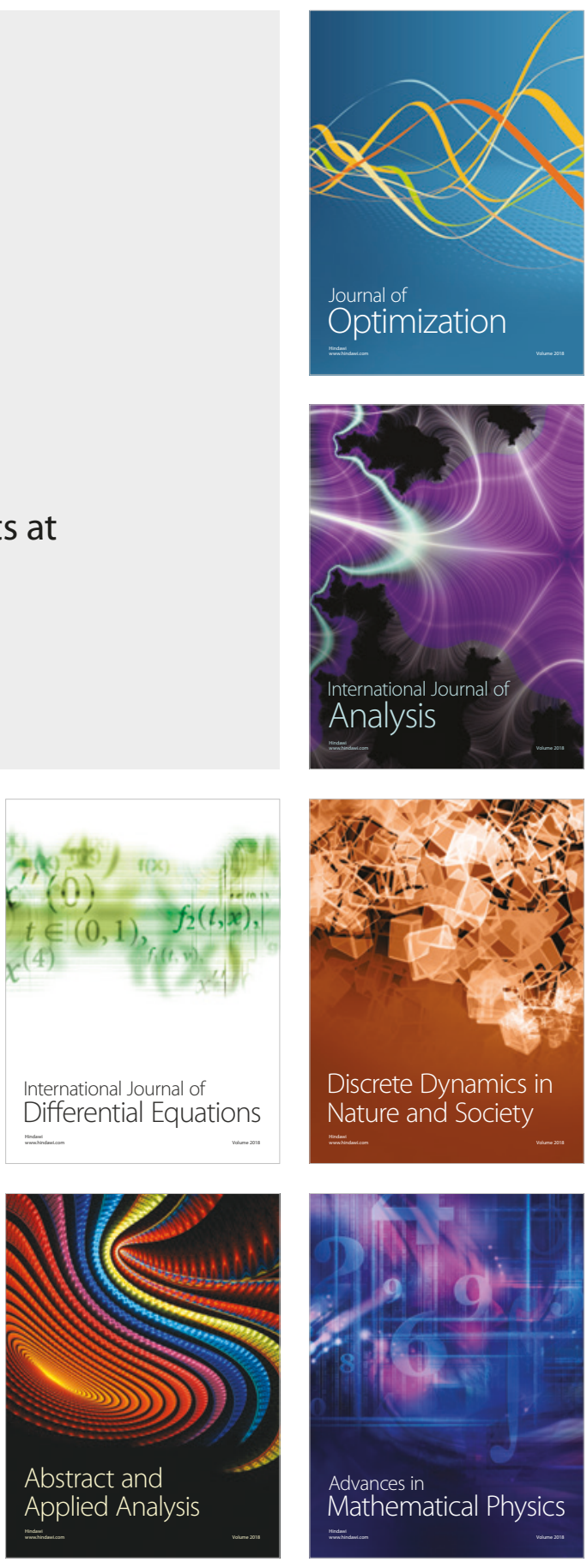